\title{
Molecular evolution of anthocyanin pigmentation genes following losses of flower color
}

\author{
Winnie W. Ho and Stacey D. Smith ${ }^{*}$
}

\begin{abstract}
Background: Phenotypic transitions, such as trait gain or loss, are predicted to carry evolutionary consequences for the genes that control their development. For example, trait losses can result in molecular decay of the pathways underlying the trait. Focusing on the lochrominae clade (Solanaceae), we examine how repeated losses of floral anthocyanin pigmentation associated with flower color transitions have affected the molecular evolution of three anthocyanin pathway genes (Chi, F3h, and Dfr).

Results: We recovered intact coding regions for the three genes in all of the lineages that have lost floral pigmentation, suggesting that molecular decay is not associated with these flower color transitions. However, two of the three genes (Chi, F3h) show significantly elevated dN/dS ratios in lineages without floral pigmentation. Maximum likelihood analyses suggest that this increase is due to relaxed constraint on anthocyanin genes in the unpigmented lineages as opposed to positive selection. Despite the increase, the values for $\mathrm{dN} / \mathrm{dS}$ in both pigmented and unpigmented lineages were consistent overall with purifying selection acting on these loci.

Conclusions: The broad conservation of anthocyanin pathway genes across lineages with and without floral anthocyanins is consistent with the growing consensus that losses of pigmentation are largely achieved by changes in gene expression as opposed to structural mutations. Moreover, this conservation maintains the potential for regain of flower color, and indicates that evolutionary losses of floral pigmentation may be readily reversible.
\end{abstract}

Keywords: Molecular decay, Reversibility, Trait evolution, Predictability, Gene fate, Evolutionary trajectory

\section{Background}

Understanding the predictability of the molecular changes associated with phenotypic transitions is a major goal in evolutionary biology. Evolutionary genetic studies across a wide range of organisms and traits have revealed that repeated phenotypic transitions are often caused by similar changes at the genetic level $[1,2]$. In some cases, this molecular convergence extends not only to the locus but also to the type of mutation and even its nucleotide position [3-5]. These patterns suggest that the causes of phenotypic evolution may often be predictable at the molecular level, given knowledge of the genetic basis for the trait [6, 7]. Indeed, for many traits, the mutations responsible for convergent phenotypes are concentrated

\footnotetext{
* Correspondence: stacey.d.smith@colorado.edu Department of Ecology and Evolutionary Biology, University of Colorado, Boulder, USA
}

(c) 2016 Ho and Smith. Open Access This article is distributed under the terms of the Creative Commons Attribution 4.0 International License (http://creativecommons.org/licenses/by/4.0/), which permits unrestricted use, distribution, and reproduction in any medium, provided you give appropriate credit to the original author(s) and the source, provide a link to the Creative Commons license, and indicate if changes were made. The Creative Commons Public Domain Dedication waiver (http://creativecommons.org/publicdomain/zero/1.0/) applies to the data made available in this article, unless otherwise stated. in a small set of "hotspot" loci [8] and are restricted to particular classes of mutations (e.g. $[9,10])$.

In addition to predictability in the mutations that cause phenotypic transitions, the genetic changes that follow phenotypic transitions can also be predictable. For example, transitions to a parasitic or symbiotic habit are often followed by pseudogenization and molecular decay in particular functional gene categories [11, 12]. Even genes which are conserved for other functions may experience an increase in evolutionary rate if the trait transition is associated with reduced breath or level of expression [13, 14]. Predictable molecular patterns are also seen at the protein level, where substitutions that cause changes in function may be followed by compensatory mutations to offset fitness declines $[15,16]$, or by mutations to optimize the derived activity and/or increase stability of the new conformation [17-20]. Despite having strong expectations about the genetic changes that are 
likely to follow many types of phenotypic transitions $[21,22]$, relatively few studies have tested these predictions at macroevolutionary scales [23, 24].

Because trait losses occur relatively frequently across the tree of life (e.g. $[25,26])$, this type of transition provides a powerful tool for understanding the predictability of genetic changes following phenotypic evolution. Trait losses may lead to a range of outcomes for genes in pathways underlying those traits. One possible outcome is the gradual erosion of pathway genes through the accumulation of inactivating mutations, leading to pseudogenization or even gene loss $[27,28]$. This process is expected to occur over the span of 0.5 to 6 million years [29] and has been documented in association with many instances of trait loss, such as the loss of eyes in cavefish [30] and teeth in edentulous mammals [31]. However, molecular decay may be prevented if the underlying genes are involved in the development of multiple traits beyond the trait that was lost. In this case, the genes may experience stabilizing selection to maintain their function or positive selection to improve function in those other contexts $[29,32]$. Another possible outcome for pathway genes following trait loss is recruitment for an entirely new function, although this pattern has never been documented. To some extent, these possible evolutionary trajectories for genes after trait loss parallel those following gene duplication, in which new gene copies may undergo pseudogenization, subfunctionalization, neofunctionalization, or escape from adaptive conflict [32-34]. Both evolutionary scenarios (trait losses and gene duplications) share a reduction in constraint that alters the selective forces experienced by genes, and consequently, their evolutionary fates. However, compared with the literature on gene duplication (reviewed in [35]), much less attention has focused on the molecular fates of single-copy genes associated with cases of trait loss.

Here we examine the molecular evolution of pigmentation pathway genes following repeated losses of floral pigmentation, focusing on the Andean clade Iochrominae in the tomato family, Solanaceae. This clade of roughly 35 species is well known for its diversity of floral colors [36], and like many other angiosperms, these colors largely derive from red, purple, and blue anthocyanin pigments [37]. These pigments are primarily produced in flowers, with little to no detectable presence in vegetative tissue in most taxa [38]. Over the estimated 5 million year history of Iochrominae [39], at least three lineages have lost floral anthocyanin pigmentation in association with transitions from the ancestral state of purple flowers to white or yellow flowers (Fig. 1, [40, 41]). The oldest loss (ca. 3 mya) is associated with a clade that has subsequently diversified to give rise to seven taxa (five shown in Fig. 1), one of which has regained pigmentation. The other two losses represent more recent events that occurred in single

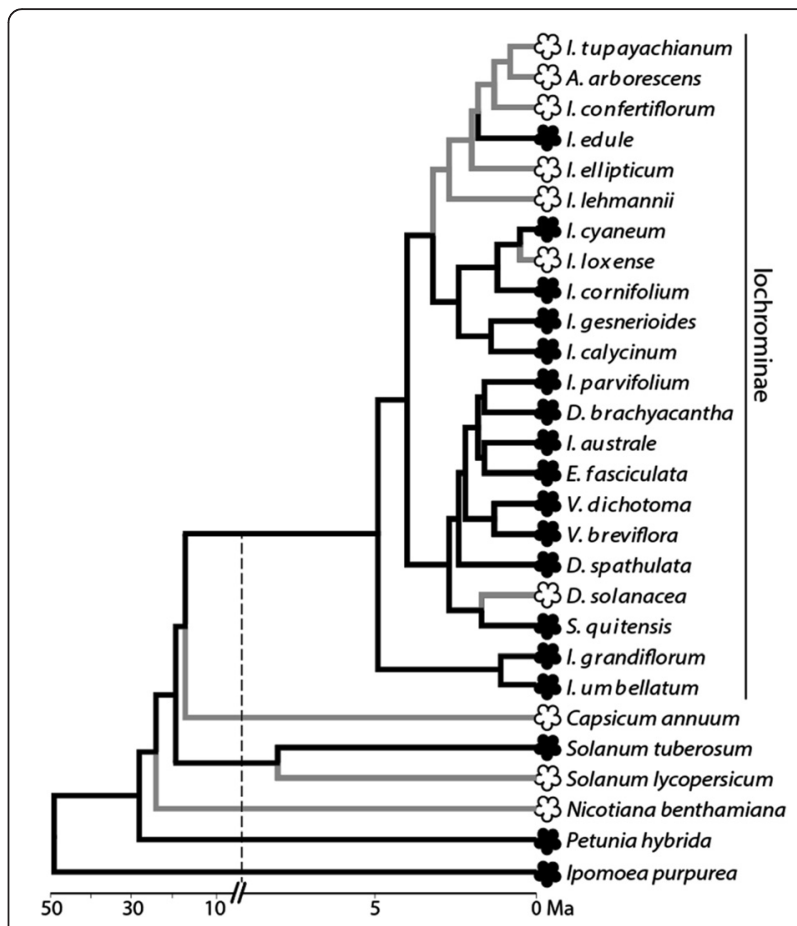

Fig. 1 Distribution of floral anthocyanin pigmentation across lochrominae. Lineages with pigmentation are represented with black flowers and black lines; those without are represented with white flowers and gray lines. Generic names within lochrominae are abbreviated as follows: $A .=$ Acnistus, D. $=$ Dunalia, E. $=$ Eriolarynx, I. = lochroma, S. = Saracha, and V. =Vassobia. Phylogeny and estimated divergence times from Sarkinen et al. [39] and Muchhala et al. [86]. Note that the timescale is split to accommodate the shallow divergences within lochrominae as well as the deeper splits among the outgroups

species (I. loxense and D. solanacea). The time span of these transitions (ca. 0.5 to 3 mya) encapsulates the range over which we would expect to see molecular consequences of changes in selective constraint on pathway genes following trait loss $[29,30]$. If selection maintained these genes primarily for their role in floral pigmentation, we expect losses of pigmentation to be followed by relaxed selective constraint and possibly pseudogenization. Alternatively, if the decay of these genes carries negative pleiotropic consequences, either for anthocyanins in other tissues or for the production of related compounds, the genes might remain under purifying selection, even in white-flowered lineages. Finally, if releasing genes from their role in floral pigmentation allows them to adopt new functions, then we might expect evidence of positive selection.

In order to test these possible effects of trait loss on molecular evolution, we focus on a set of three anthocyanin pathway genes, Chi (chalcone isomerase), F3h (flavanone-3-hydroxylase), and Dfr (dihydroflavonol4-reductase). The $D f r$ gene represents the most downstream step among these three, and it appears to be exclusively involved in anthocyanin biosynthesis in 
Solanaceae ([42], Fig. 2). Thus, it might be predicted to experience the strongest effects of relaxed constraint following loss of floral anthocyanins. By contrast, the other two upstream enzymes are required for the production of multiple flavonoids, including anthocyanins, flavones, and flavonols (Fig. 2). The uncolored flavones and flavonols are primarily involved in responses to UV stress [43, 44], but also play a role in male fertility and signaling in some species [45, 46]. Thus, Chi and F3h might remain under purifying selection despite the loss of floral pigmentation because of their pathway position and functional significance. The dynamics of Chi evolution may also be influenced by the fact that, unlike the single copy $D f r$ and $F 3 h$, this enzyme is encoded by two loci (Chi-A, the principally active copy, and Chi-B, only expressed in young anthers) $[47,48]$. In this analysis, we focus on the Chi-A copy (hereafter ' $C h i$ ') that is required for floral pigmentation in Solanaceae $[48,49]$. The extent to which these genes experience relaxed selective pressures following the loss of floral pigmentation has important consequences

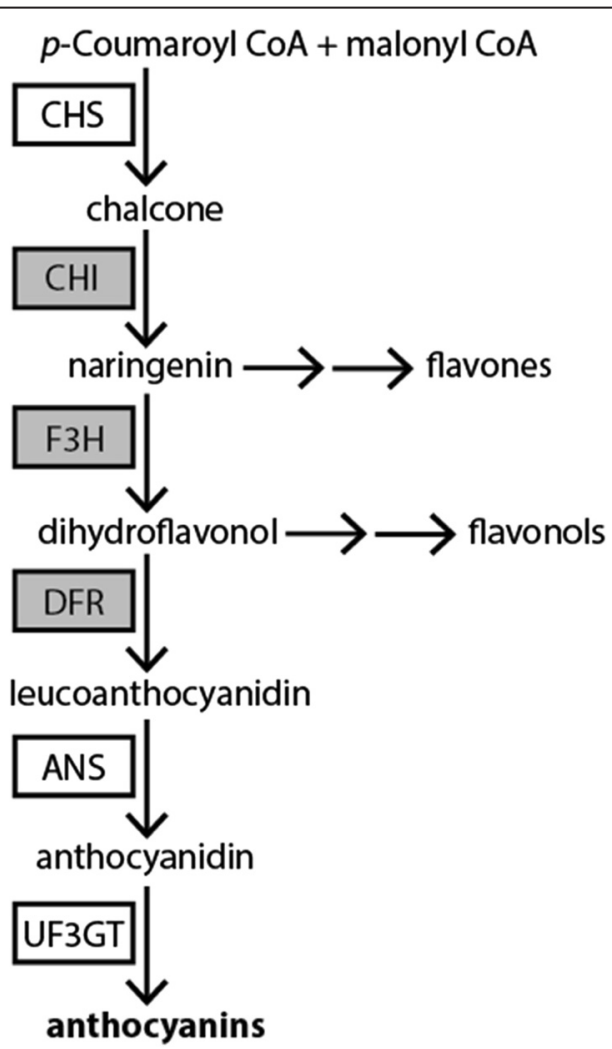

Fig. 2 Core anthocyanin biosynthetic pathway adapted from Rausher [56]. Enzymes include: CHS, chalcone synthase; $\mathrm{CHI}$, chalcone isomerase; F3H, flavanone-3-hydroxylase; DFR, dihydroflavonol reductase; ANS, anthocyanidin synthase; and UF3GT, UDP-glucose flavonoid 3-O-glucosyl transferase. Several of the steps required for anthocyanin pigment production are shared with other uncolored flavonoid compounds (e.g., flavones and flavonols). The enzymes examined in this study are shaded in gray for the evolutionary trajectory of these plant lineages, as the molecular decay of any of these loci would significantly reduce the potential for future regain of this trait.

\section{Results}

Sequence variation in Chi, F3h, and Dfr across lochrominae

We recovered complete coding regions for the three anthocyanin pathway genes Chi (717 bp), F3h (1113 bp), and $D f r(1179 \mathrm{bp})$ from all of the sampled pigmented and unpigmented Iochrominae (Fig. 1). Within Iochrominae, we observed no length variation (insertion-deletion events). Across all loci, pairwise divergence was low, with less than $3 \%$ difference at the nucleotide level and less than $1.5 \%$ at the amino acid level across Iochrominae. Divergence was higher at the family level, ranging from $8 \%$ at the amino acid level in Dfr across Solanaceae to $20 \%$ in Chi, the most rapidly evolving of the three genes (Additional file 1: Figure S1). However, we did not observe any clear inactivating mutations, such as premature stop codons, or frameshifts, in any of the sequences (Additional file 2: Table S1).

\section{Variation in selective constraint across genes and across lineages}

We applied codon-based maximum likelihood methods to characterize patterns of molecular evolution across these loci and test for the effect of pigment loss on selective constraint. Models with a single ratio of nonsynonymous to synonymous substitution rates $(\mathrm{dN} / \mathrm{dS}$ or $\omega)$ for each locus resulted in values between 0.09 and 0.24 , suggesting that these loci have predominantly experienced purifying selection (Additional file 3: Table S2a, Fig. 3). Among the three loci, Chi has the highest $\mathrm{dN} / \mathrm{dS}$ ratio, followed by $D f r$ and F3h. The values for $C h i$ and $D f r$ are statistically indistinguishable, while that for F3h is significantly lower (Additional file 4: Table S3, Fig. 3). The range of $d N / d S$ ratios for these genes in our study is similar to that found for anthocyanin transcription factors and other core anthocyanin pathway genes in previous studies [50,51].

We next estimated branch models with separate $\mathrm{dN} /$ dS ratios for pigmented and unpigmented lineages in order to test the hypothesis that these flower color transitions have altered the selective pressures acting on pigmentation genes. The two-ratio models revealed higher $\mathrm{dN} / \mathrm{dS}$ ratios in unpigmented lineages for all of the loci (Fig. 3), consistent with relaxed selective constraint. The ratio for unpigmented lineages was nearly twice as high as that for pigmented lineages in both Chi $(0.377$ vs. 0.185$)$ and F3h (0.128 vs. 0.0792), and the two ratio models for these genes resulted in significantly higher likelihoods than the single ratio models (Additional file 3: Table S2a). Although $\mathrm{dN} / \mathrm{dS}$ is slightly elevated in 


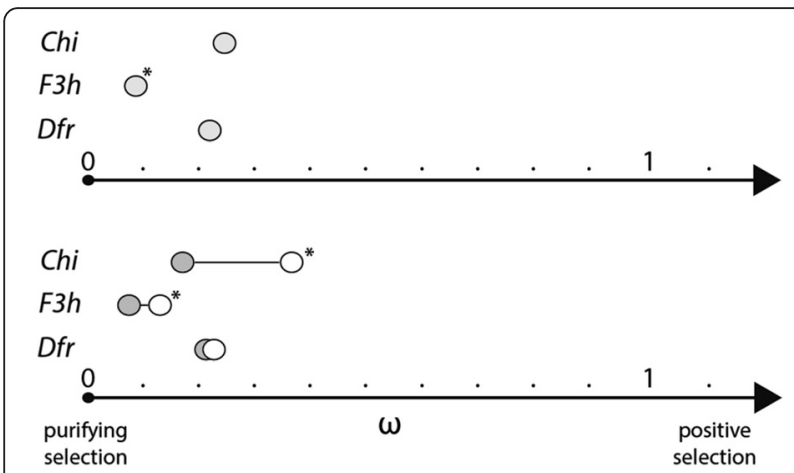

Fig. 3 Patterns of variation in the $\mathrm{dN} / \mathrm{d} S$ ratio $(\omega)$ across genes and between pigmented and unpigmented lineages. The upper panel shows $\omega$ values for the single ratio model for each of the three loci (see also Additional file 4: Table S3). * represents a locus with a significantly lower $\omega$ value than the other two loci. The lower panel shows $\omega$ values for pigmented (dark gray dot) and unpigmented (white dot) lineages under the two-ratio model. * represents cases in which the unpigmented lineages have significantly higher $\omega$ values

unpigmented lineages for $D f r$, a single ratio model could not be rejected (Additional file 3: Table S2a).

Considering that the timing of losses of floral pigmentation varies across the sampled taxa (Fig. 1), we examined two additional models allowing for different ratios among the unpigmented lineages. In the first of these, we divided the unpigmented lineages between those in the outgroups (three taxa) versus those in the ingroup (seven taxa). We found the same pattern as in the two ratio models; the unpigmented lineages in both the ingroup and outgroup have elevated $\mathrm{dN} / \mathrm{dS}$ ratios relative to the background ratio in the pigmented lineages for both Chi and F3h. However, this division did not represent a significant improvement over the two ratio models (Additional file 3: Table S2a). We also tested models separating the clade which has diversified since a recent loss (comprising I. tupayachianum, A. arborescens, and I. confertiflorum) from the remaining unpigmented lineages and recovered the same result (elevated $\mathrm{dN} / \mathrm{dS}$ ratios in unpigmented lineages but no improvement in likelihood over the two ratio models). These analyses suggest that the pattern of elevated $\mathrm{dN} / \mathrm{dS}$ ratios following losses of floral anthocyanins is not taxonspecific (e.g., only in Iochrominae) but rather shared across all of the sampled lineages without floral anthocyanins.

\section{Testing for positive selection in unpigmented lineages}

The finding of elevated $\mathrm{dN} / \mathrm{dS}$ ratios following losses of floral pigmentation could be attributed either to reduced selective constraint or positive selection acting on multiple sites across the genes. We implemented an additional series of maximum likelihood models to distinguish among these explanations. First, we fit alternative branchsite models to test the hypothesis that a proportion of sites within the unpigmented lineages has experienced positive selection ( $\mathrm{dN} / \mathrm{dS}$ greater than 1$)$. In these comparisons, we found no evidence of positively selected sites in the unpigmented lineages (Additional file 3: Table S2b). The majority of the sites (66 to $88 \%$ depending on the locus) are inferred to have experienced purifying selection in both pigmented and unpigmented lineages while the remainder are evolving neutrally in one or both (Additional file 3: Table S2b). As additional confirmation of this pattern, we fit simpler sites models (in which the categories are not partitioned across pigmented and unpigmented branches), and these analyses were also consistent with a lack of positive selection. Models including a proportion of sites under positive selection did not result in a significantly higher likelihood for Chi or F3h (Additional file 3: Table S2c). Although the addition of a proportion of positively selected sites did significantly improve the likelihood for $D f r$, no positively selected sites were identified in the using the Bayes Empirical Bayes analysis $(p>0.95)$ (Additional file 3: Table S2c). Given previous work on $D f r$, we suspect the better fit of models with a category of positively selected sites reflects selection associated with shifts among different pigment types (e.g., blue and red: [19]) as opposed to gains and losses of pigments.

Because the $\mathrm{dN} / \mathrm{dS}$ based tests may have low power when selection has not acted repeatedly on the same sites [52], we employed alternative methods that focus on physiochemical properties [53]. Unlike $\mathrm{dN} / \mathrm{dS}$ based tests, which treat all non-synonymous substitutions equally, these methods consider the physiochemical effects of amino acid changes and the magnitude of their predicted impacts on protein function. Positively selected sites are identified by comparison with a expected distribution of effects based on codon frequencies [54]. Focusing on the unpigmented lineages, we found zero substitutions with significant physiochemical effects in F3h (Additional file 5: Table S4). Only one such substitution was identified in $D f r$, and this change occurred in one of the two unpigmented outgroup taxa (Additional file 5: Table S4). We found six sites with non-conservative substitutions in unpigmented lineages in Chi (Additional file 5: Table S4) although a far greater number (22 total) were found among the pigmented lineages (Additional file 5: Table S4). Thus, the proportion of radical changes on unpigmented lineages (6 of 28 , or 0.21 ) is less than the proportion of unpigmented branches in the phylogeny (15 of 54, or 0.28 ). These patterns suggest that positive selection is not elevated in the unpigmented lineages compared to pigmented ones, but that overall, Chi has experienced a more dynamic evolutionary history than the other two loci, as evidenced by its longer branch lengths (Additional file 1: Figure S1).

\section{Discussion}

Following evolutionary losses of floral anthocyanin production, we predicted a range of possible outcomes for 
genes in the anthocyanin pathway. On one extreme, if the role of these genes was limited to flower color, we would predict that losses of floral pigmentation would lead to relaxed constraint and possibly pseudogenization. However, to the extent that these genes are important for traits beyond flower color, we might expect them to experience purifying selection, even in lineages without floral anthocyanins. In addition, the genes could undergo positive selection to optimize their non-floral functions or to acquire new functions in lineages which no longer produce floral anthocyanins. Our results indicated that all three of the pathway genes remain under purifying selection and have not experienced positive selection following multiple losses of pigmentation across the phylogeny. While the ratio of non-synonymous to synonymous substitutions was elevated in lineages without floral anthocyanins, we did not find evidence for positive selection, as would have been anticipated if the genes had shifted to new functions. These results suggest that while losses of flower color relax the level of purifying selection acting on pigment genes, they do not lead to irreversible molecular decay.

\section{Conservation of anthocyanin pathway genes}

Our study supports previous findings that the core genes of the anthocyanin pathway are highly conserved, likely due to their wide range of functions in plant physiology. Anthocyanins and related flavonoids are found across all land plants and thus, the structural elements of the pathway trace back to their common ancestor over 400 million years ago $[55,56]$. Depending on the taxon, these compounds may play a role in pigmentation, UV stress response, signaling, and defense [37, 43, 46]. Accordingly, a loss of function of any of the core genes of the pathway (Fig. 2) would likely affect many aspects of plant fitness. Consistent with this hypothesis, we found that Chi, F3h, and Dfr have remained conserved through multiple rounds of flower color loss at varying timescales (Fig. 1). All of the white and yellow flowered lineages presented complete coding regions, without premature stop codons or frameshift mutations, suggesting that these copies have retained their function. Although additional experiments would be required to confirm their function (e.g. [21, 57]), the presence of uncolored flavonoids (e.g., flavonols and flavones, Fig. 2) in the flowers of several of the unpigmented taxa $[38,58]$ supports the conclusion that Chi and F3h have been conserved. The factors responsible for the conservation of $D f r$ are less clear as it is only known to function in anthocyanin biosynthesis in Solanaceae [42] and anthocyanins have thus far not been detected in non-floral tissues in the white and yellow-flowered lineages of Iochrominae [38]. One possibility is that $D f r$ contributes to pigment production in these lineages but only under particular conditions (e.g., drought or heat stress; [43]). Alternately, this enzyme may participate in additional biochemical reactions beyond those presently identified in the literature (Fig. 2).

Although this represents the one of the few systematic studies of anthocyanin genes across a clade (see also [50]), the strong conservation of Chi, F3h, and Dfr is consistent with findings from population level studies. Decades of research on flower color variation in natural populations have failed to uncover any segregating loss-of-function mutations at Chi or F3h and only two for $D f r[59,60]$. Moreover, the individuals carrying the known $D$ fr mutations in both cases are exceptionally rare relative to their pigmented counterparts. Given that such loss-of-function mutations are known to be quite common among horticultural varieties, these patterns at micro and macroevolutionary scales suggest that selection strongly disfavors mutations which disrupt the function of any of the core genes, preventing their fixation [51].

The conservation of these core genes stands in contrast to other elements of the larger flavonoid pathway, which have the potential to rapidly pseudogenize. In particular, the $F 3^{\prime} 5$ ' $h$ gene, which hydroxylates dihydroflavonols to produce the precursors of blue anthocyanins, has been found to repeatedly experience pseudogenization or deletion following transitions to red flower colors $[61,62]$. In both well-studied cases, these color transitions have been associated with floral radiations occurring on the order of 1 to 2 million years ago [36, 39, 63]. Compared to the core genes examined in this study, which are required for production of anthocyanins and many other flavonoids, the role of $F 3^{\prime} 5^{\prime} h$ appears to be limited to the blue anthocyanins, lessening the potential pleiotropic effect of its loss [21]. This contrast between the evolutionary history of $F 3^{\prime} 5^{\prime} h$ and the core genes examined here suggests that the timespan of the Iochrominae radiation (ca. 5 million years) would be sufficient to observe pseudogenization of Chi, F3h, or Dfr if these genes were not maintained by selection.

\section{Relaxed constraint following losses of floral pigmentation} Although the core genes have been conserved in lineages without floral anthocyanins, our analyses indicate that $C h i$ and $F 3 h$ have experienced altered selection pressures. Models allowing different $\mathrm{dN} / \mathrm{dS}$ ratios for pigmented and unpigmented branches of the phylogeny resulted in a significantly better fit to the data (Additional file 3: Table S2a), and in both cases, the ratio for the unpigmented branches was roughly twice the ratio for the pigmented branches (Fig. 3). Even with this increase, all of the $\mathrm{dN} / \mathrm{dS}$ values fall into the range expected for genes that are under purifying selection (i.e., less than 1). For $D f r$, we also found an increase in $\mathrm{dN} / \mathrm{dS}$ in unpigmented lineages, 
but the addition of this extra parameter did not improve the likelihood (Additional file 3: Table S2a).

While such an elevated $\mathrm{dN} / \mathrm{dS}$ could be due to the action of positive selection, our subsequent analyses suggest that instead the pattern is due to relaxed selective constraint. Branch-site models that allow a proportion of sites to experience positive selection $(\mathrm{dN} / \mathrm{dS}>1)$ did not provide a better fit to the data and identified no positively selected sites in unpigmented lineages $(\mathrm{PP}>0.95)$. Given that these analyses do not account for the potential functional consequences of mutations, we compared these results to an alternative physiochemical approach to identifying positive selection [53]. These analyses found little evidence of radical amino acid substitutions in Dfr and F3h, and none within the white or yellow flowered Iochrominae. While Chi experienced a larger number of radical changes, most of these occurred in pigmented lineages, and in particular, in the long branches of the outgroups (Additional file 5: Table S4). Thus, although some functional evolution has likely occurred across the phylogeny, these analyses do not implicate losses of flower pigmentation in driving these changes.

As with our study, many instances of trait loss have been followed by relaxed selection on the underlying genes (e.g. [30, 64]), while positive selection on these genes has never been reported. Theoretically, once a gene is no longer required for a particular task, it has the potential to be co-opted for a new function, in a similar fashion to what has been observed for duplicate copies of genes (e.g., [32, 65, 66]). One explanation for the lack of empirical examples may simply be the relative paucity of traits for which the genetic basis is sufficiently well known to test the effects of trait loss on molecular evolution of the underlying pathway. We suspect that cases may be uncovered as our knowledge of the genotype-phenotype map expands to include a wider array of traits and taxa.

\section{Pathway position and rate variation}

Studies of biochemical pathways have shown that properties of the interacting genes often predict patterns of molecular evolution. For example, more highly connected enzymes (e.g., those share more substrates or products with other enzymes) tend to evolve more slowly than those which are less connected ([67], but see [68]). In the case of the anthocyanin pathway, the upstream genes have been predicted to experience stronger evolutionary constraint than downstream genes because their control on flux through the pathway and because of the number of products that require their activity [69]. Indeed, previous studies of anthocyanin genes have found evidence for this positional effect, where upstream genes generally evolve more slowly due to differences in the selective constraints $[50,69,70]$.
While our study found significant variation in rates of evolution across the genes, we did not find that most upstream genes experienced the highest constraint. Chi was the most rapidly evolving gene with the highest $\mathrm{dN} / \mathrm{dS}$ ratio among the three (Fig. 3, Additional file 1: Figure S1) despite the fact that it occupies the most upstream position. Partitioned analyses of the three gene dataset showed that the $\mathrm{dN} / \mathrm{dS}$ ratio for $C h i$ is significantly higher than for $F 3 h$, and statistically indistinguishable from that for $D f r$ (Additional file 4: Table S3). This high rate of evolution for Chi may be related to several unique aspects of its function and evolutionary history. First, unlike the other two loci, Chi has at least two closely related duplicates $[47,48]$, which may allow for reduced constraint on the individual copies. Second, the reaction catalyzed by $\mathrm{CHI}$, namely the cyclization of chalcone into naringenin, can occur spontaneously (although inefficiently) and thus is likely to occur in cells where CHS is active even if $\mathrm{CHI}$ is not present [71, 72]. These factors may help to explain why $C h i$ evolves as quickly as the downstream genes like $D f r$, a result also found in Rausher et al. [69]. Future studies examining the full pathway (Fig. 2) would clarify the extent to which the pathway position predicts rate variation and whether indeed Chi is a strong outlier among upstream genes.

\section{Conclusions}

On a phylogenetic scale, trait losses have commonly been associated with molecular decay of the underlying pathway [27, 30, 73]. However, such decay is expected to be mitigated when the genes of the pathway are required for multiple functions [74]. In the case of floral anthocyanin pigmentation, our study shows that the core genes of the anthocyanin pigmentation pathway have been conserved through repeated losses of floral pigmentation, a result that likely reflects the importance of these genes for functions beyond flower color. The conservation of these genes preserves the potential for regain of flower color following loss as observed in Iochroma (Fig. 2) and other flowering plant clades [41].

Despite this overall structural conservation, we identify patterns consistent with relaxed selective constraint in two of the three pathway genes (Chi and F3h) following losses of floral pigmentation. Given that the upstream products of these two genes, flavones and flavonols, are often produced in flowers and leaves [38], the elevated $\mathrm{dN} / \mathrm{dS}$ ratios we observe are not likely to indicate a decrease in functionality of the enzymes. Instead, this molecular evolutionary pattern may be a consequence of changes in the expression of these genes. Specifically, reduced gene expression is well known to result in higher rates of protein evolution due to relaxed selection for translational efficiency and robustness [75, 76]. Although it is not known whether losses of floral 
pigmentation in Iochrominae are associated with reduced expression of Chi and F3h, such downregulation has been documented in other taxa [77]. Ongoing studies in the Iochrominae system aimed at integrating analyses of molecular evolution, gene expression, and flavonoid production along the phylogeny will contribute to a better understanding of both the causes and consequences of phenotypic transitions.

\section{Methods}

\section{Dataset construction}

We sequenced Chi-A, F3h, and Dfr from a total of 22 Iochrominae species (shown in Fig. 1). These included all 7 species that lack floral anthocyanin pigmentation and 15 species that produce floral anthocyanins. The genes were amplified from floral bud cDNA prepared from greenhouse or field-collected material. Briefly, RNA was extracted using the Spectrum Total RNA extraction kit (Sigma-Aldrich, MO), and DNA removed using an oncolumn DNAse digestion (Qiagen, Netherlands). cDNA synthesis was carried out using lug of RNA and the SuperScriptII Reverse Transcription kit (Life Technologies, CA). Chi, F3h, and $D f r$ were amplified using primers designed from previous studies $[59,61]$. Products were directly sequenced in both directions, assembled, and edited using Geneious 7.1.5. Voucher specimens and accession numbers for all sequences are provided in Additional file 2: Table S1. Sequences from six outgroup taxa were retrieved from Genbank or Solgenomics (solgenomics.net); these included five from across Solanaceae (Nicotiana benthamiana, Solanum tuberosum, S. lycopersicum, Capsicum annuum and Petunia hybrida) and one from the sister family Convolvulaceae (Ipomoea purpurea) (Fig. 1, Additional file 2: Table S1).

\section{Estimating rates of amino acid substitution}

Codon-based maximum likelihood methods were implemented using the codeml program in PAML4.7a [78]. These methods estimate the strength and nature of selection acting on codon sites by calculating the ratio of non-synonymous to synonymous mutations ( $\mathrm{dN} / \mathrm{dS}$ or $\omega$ ), where $\omega<1$ suggests purifying selection, $\omega=1$ indicates neutral evolution, and $\omega>1$ indicates positive selection. Variation in $\omega$ can be partitioned across genes, across sites within a gene, and across branches to test specific hypotheses. We first examined the hypothesis that flower color transitions (specifically losses of floral anthocyanins) have altered selective pressures on anthocyanin genes using branch models. These models allow for $\omega$ to differ between background (pigmented) and foreground (unpigmented) branches [79]. For significance testing, the two ratio branch models for each gene were compared to a single ratio model using likelihood ratio tests. We also considered branch models in which we divided the unpigmented lineages into two sets (e.g., separate $\omega$ values for unpigmented lineages in the ingroup versus the outgroup), and used the same likelihood ratio tests for model comparison. Observing that the $\omega$ values from the single ratio models varied across genes, we conducted an additional set of analyses, grouping the genes into all possible pairs to test differences in $\omega$ across the genes (Models "C" and "E", [80]).

Within the same maximum likelihood framework, we used branch-site models to test for positive selection acting on the anthocyanin genes in unpigmented lineages. For these analyses, the null model assumes four site classes [81]. Class 0 includes sites that are conserved $(0<\omega<1)$ across all branches. Class 1 includes sites that evolve neutrally $(\omega=1)$ across all branches. Class $2 \mathrm{a}$ contains sites that are conserved in the background, but become neutral in the foreground. Class $2 \mathrm{~b}$ contains sites that evolve neutrally in the background, and are restricted to neutral in the foreground. The alternative model is similar, but allows for positive selection by permitting $\omega$ to vary freely in the foreground branches of classes $2 \mathrm{a}$ and $2 \mathrm{~b}$. These branch-site models also include a Bayes Empirical Bayes (BEB) procedure for the identification of sites under positive selection [52]. We considered sites to be positively selected if the posterior probability was greater than 0.95 .

As the branch-site models are relatively parameterrich, we also fit sites models for each gene, which are designed to detect positively selected sites across the entire tree. We conducted two sets of model comparisons for each gene, M1a versus M2a and M7 versus M8 [52, 82]. The M1a model has two site classes, one conserved $(0<\omega<1)$ and one neutral $(\omega=1)$, while the M2a model has an extra category of positively selected sites $(\omega>1)$. The M7 model describes variation in $\omega$ as a beta distribution between 0 and 1 with parameters estimated from the data. The distribution is discretized into 10 equally spaced categories [52]. The M8 model adds an extra site category where positive selection is permitted $(\omega \geq 1)$.

\section{Analysis of physiochemical changes following losses of pigmentation}

As a alternative approach to detecting changes in selection pressure associated with losses of pigmentation, we used the program TreeSAAP 3.2 [53] to examine the physiochemical effects of amino acid substitutions along unpigmented branches. TreeSAAP identifies positively selected sites by comparing the magnitude of the observed physiochemical changes (reconstructed along branches with PAML) to an expected distribution assuming random amino acid replacements [54]. The program examines 31 physiochemical properties, ranging from polarity to molecular weight, which are 
important for determining the structure and function of the protein. For each property, the effect of a substitution is assigned to a category from 1 to 8 , with categories 6 through 8 being considered radical. Significance is determined by a goodness-of-fit test comparing the distribution of observed and expected effects [83]. For these analyses, we used a sliding window length of 20 sites and considered only sites with effects in the 6 to 8 magnitude range as radical. We examined only substitutions within in the portion of the protein known to contribute to its three-dimensional structure. To do this, Solanaceae sequences were aligned with known crystal structures for DFR [84] and CHI [72], viewed in the Swiss PDB viewer [85].

\section{Ethics}

Not applicable.

\section{Consent to publish}

Not applicable.

\section{Availability of data and materials}

All sequence data has been submitted to Genbank via the following accession numbers: KT898394-KT898451. Sequence alignments have been placed on Dryad at doi:10.5061/dryad.49rs8.

\section{Additional files}

Additional file 1: Figure S1. Gene trees for Chi, F3h and Dfr. (DOCX $88 \mathrm{~kb}$ ) Additional file 2: Table S1. Source information and Genbank numbers for sequences. (DOCX $25 \mathrm{~kb}$ )

Additional file 3: Table S2. Maximum likelihood tests of selection on Chi, F3h, and Dfr. (DOCX $26 \mathrm{~kb}$ )

Additional file 4: Table S3. Comparisons of $d N / d S$ across genes, on Chi, F3h, and Dfr. (DOCX $15 \mathrm{~kb}$ )

Additional file 5: Table S4. Amino acid substitutions with significant physiochemical effects along unpigmented and pigmented branches. (DOCX $28 \mathrm{~kb}$ )

\section{Abbreviations}

$\mathrm{CHI}$ : chalcone isomerase; DFR: dihydroflavonol reductase; F3H: flavanone-3-hydroxylase.

\section{Competing interests}

The authors declare that they have no competing interests.

\section{Authors' contributions}

WWH and SDS contributed to data collection and analysis, experimental design, and writing and revising the manuscript. All authors have read and approved of the final version of the manuscript.

\section{Acknowledgements}

We thank Jeff Neiman for assistance with data collection and molecular work.

\section{Funding}

This work was funded by NSF DEB 1355518 (SDS).

Received: 28 January 2016 Accepted: 29 April 2016

Published online: 10 May 2016

\section{References}

1. Arendt J, Reznick D. Convergence and parallelism reconsidered: what have we learned about the genetics of adaptation? Trends Ecol Evolut. 2008;23:26-32.

2. Manceau M, Domingues VS, Linnen CR, Rosenblum EB, Hoekstra HE. Convergence in pigmentation at multiple levels: mutations, genes and function. Philos Trans R Soc B Biol Sci. 2010;365:2439-50.

3. McCracken KG, Barger CP, Bulgarella M, Johnson KP, Sonsthagen SA, Trucco J, Valqui TH, Wilson RE, Winker K, Sorenson MD. Parallel evolution in the major haemoglobin genes of eight species of Andean waterfowl. Mol Ecol. 2009;18:3992-4005.

4. Reed RD, Papa R, Martin A, Hines HM, Counterman BA, Pardo-Diaz C, Jiggins CD, Chamberlain NL, Kronforst MR, Chen R, Halder G. Optix drives the repeated convergent evolution of butterfly wing pattern mimicry. Science. 2011:333(6046):1137-41.

5. Shapiro MD, Bell MA, Kingsley DM. Parallel genetic origins of pelvic reduction in vertebrates. Proc Natl Acad Sci. 2006;103:13753-8.

6. Gompel N, Prud'homme B. The causes of repeated genetic evolution. Dev Biol. 2009:332:36-47.

7. Stern DL, Orgogozo V. The loci of evolution: how predictable is genetic evolution? Evolution. 2008:62:2155-77.

8. Martin A, Orgogozo V. The loci of repeated evolution: a catalog of genetic hotspots of phenotypic variation. Evolution. 2013;67:1235-50.

9. Sucena E, Delon I, Jones I, Payre F, Stern DL. Regulatory evolution of shavenbaby/ovo underlies multiple cases of morphological parallelism. Nature. 2003;424:935-8.

10. Wray GA. The evolutionary significance of cis-regulatory mutations. Nat Rev Genet. 2007:8:206-16

11. Burke GR, Moran NA. Massive genomic decay in Serratia symbiotica, a recently evolved symbiont of aphids. Genome Biol Evol. 2011;3:195-208.

12. Palmer JD. Loss of photosynthetic and chlororespiratory genes from the plastid genome of a parasitic flowering plant. Nature. 1990;348:337-9.

13. Drummond DA, Bloom JD, Adami C, Wilke CO, Arnold FH. Why highly expressed proteins evolve slowly. Proc Natl Acad Sci U S A. 2005: 102(40):14338-43

14. Duret L, Mouchiroud D. Determinants of substitution rates in mammalian genes: expression pattern affects selection intensity but not mutation rate. Mol Biol Evol. 2000;17(1):68-070.

15. Lunzer M, Golding GB, Dean AM. Pervasive cryptic epistasis in molecular evolution. PLoS Genet. 2010; doi: 10.1371/journal.pgen.1001162.

16. Poon $\mathrm{A}$, Chao $\mathrm{L}$. The rate of compensatory mutation in the DNA bacteriophage $\varphi$ X174. Genetics. 2005;170:989-99.

17. Bridgham JT, Ortlund EA, Thornton JW. An epistatic ratchet constrains the direction of glucocorticoid receptor evolution. Nature. 2009;461:515-9.

18. Bridgham JT, Keay J, Ortlund EA, Thornton JW. Vestigialization of an allosteric switch: Genetic and structural mechanisms for the evolution of constitutive activity in a steroid hormone receptor. PLoS Genet. 2014; doi:10.1371/journal.pgen.1004058.

19. Smith SD, Wang S, Rausher MD. Functional evolution of an anthocyanin pathway enzyme during a flower color transition. Mol Biol Evol. 2013; 30:602-12.

20. Weinreich DM, Delaney NF, DePristo MA, Hartl DL. Darwinian evolution can follow only very few mutational paths to fitter proteins. Science. 2006;312:111-4.

21. Wessinger CA, Rausher MD. Lessons from flower colour evolution on targets of selection. J Exp Bot. 2012;63:5741-9.

22. Wright SI, Ness RW, Foxe JP, Barrett SC. Genomic consequences of outcrossing and selfing in plants. Int J Plant Sci. 2008;169:105-18.

23. Hersch-Green El, Myburg H, Johnson MT. Adaptive molecular evolution of a defence gene in sexual but not functionally asexual evening primroses. J Evol Biol. 2012;25:1576-86.

24. Ross-lbarra J. Genome size and recombination in angiosperms: a second look. J Evol Biol. 2007;20:800-6.

25. Porter ML, Crandall KA. Lost along the way: the significance of evolution in reverse. Trends Ecol Evol. 2003;18:541-7.

26. Wiens JJ. Widespread loss of sexually selected traits: how the peacock lost its spots. Trends Ecol Evol. 2001;16:517-23.

27. Cui J, Pan YH, Zhang Y, Jones G, Zhang S. Progressive pseudogenization: vitamin C synthesis and its loss in bats. Mol Biol Evol. 2011;28:1025-31.

28. Wickett NJ, Fan Y, Lewis PO, Goffinet B. Distribution and evolution of pseudogenes, gene losses, and a gene rearrangement in the plastid 
genome of the nonphotosynthetic liverwort, Aneura mirabilis (Metzgeriales, Jungermanniopsida). J Mol Evol. 2008;67:111-22.

29. Marshall CR, Raff EC, Raff RA. Dollo's law and the death and resurrection of genes. Proc Natl Acad Sci. 1994;91:12283-7.

30. Niemiller ML, Fitzpatrick BM, Shah P, Schmitz L, Near TJ. Evidence for repeated loss of selective constraint in rhodopsin of amblyopsid cavefishes (Teleostei: Amblyopsidae). Evolution. 2013;67:732-48.

31. Meredith RW, Gatesy J, Murphy WJ, Ryder OA, Springer MS. Molecular decay of the tooth gene enamelin (ENAM) mirrors the loss of enamel in the fossil record of placental mammals. PLoS Genet. 2009; doi:10.1371/journal.pgen.1000634.

32. Des Marais DL, Rausher MD. Escape from adaptive conflict after duplication in an anthocyanin pathway gene. Nature. 2008;454:762-5.

33. Hahn MW. Distinguishing among evolutionary models for the maintenance of gene duplicates. J Hered. 2009;100:605-17.

34. Ohno S. Evolution by gene duplication. New York: Springer Science \& Business Media; 1970.

35. Taylor JS, Raes J. Duplication and divergence: the evolution of new genes and old ideas. Annu Rev Genet. 2004;38:615-43.

36. Smith SD, Baum DA. Phylogenetics of the florally diverse Andean clade lochrominae (Solanaceae). Am J Bot. 2006;93(8):1140-53.

37. Winkel-Shirley B. Flavonoid biosynthesis. A colorful model for genetics, biochemistry, cell biology, and biotechnology. Plant Physiol. 2001;126:485-93.

38. Berardi AE, Hildreth SB, Helm RF, Winkel BS, Smith SD. Evolutionary correlations in flavonoid production across flowers and leaves in the lochrominae (Solanaceae). Phytochemistry (in revision).

39. Särkinen T, Bohs L, Olmstead RG, Knapp S. A phylogenetic framework for evolutionary study of the nightshades (Solanaceae): a dated 1000-tip tree. BMC Evol Biol. 2013;13:214

40. Smith SD, Baum DA. Systematics of lochrominae (Solanaceae): patterns in floral diversity and interspecific crossability. Acta Horticulturae. 2007;745:241-54.

41. Smith SD, Goldberg EE. Tempo and mode of flower color evolution. Am J Bot. 2015;102(7):1014-25.

42. Eich E. Solanaceae and Convolvulaceae: Secondary metabolites: Biosynthesis, chemotaxonomy, biological and economic significance (a handbook). Berlin: Springer Science \& Business Media; 2008.

43. Chalker-Scott L. Environmental significance of anthocyanins in plant stress responses. Photochem Photobiol. 1999;70:1-9.

44. Ryan KG, Swinny EE, Markham KR, Winefield C. Flavonoid gene expression and UV photoprotection in transgenic and mutant Petunia leaves. Phytochemistry. 2002;59(1):23-32.

45. Mo Y, Nagel C, Taylor LP. Biochemical complementation of chalcone synthase mutants defines a role for flavonols in functional pollen. Proc Natl Acad Sci. 1992;89(15):7213-7.

46. Shirley BW. Flavonoid biosynthesis:'new'functions for an 'old'pathway. Trends Plant Sci. 1996;1:377-82

47. De Jong WS, Eannetta NT, De Jong DM, Bodis M. Candidate gene analysis of anthocyanin pigmentation loci in the Solanaceae. Theor Appl Genet. 2004; 108:423-32.

48. Van Tunen AJ, Koes RE, Spelt CE, Van der Krol AR, Stuitje AR, Mol JN. Cloning of the two chalcone flavanone isomerase genes from Petunia hybrida: coordinate, light-regulated and differential expression of flavonoid genes. EMBO J. 1988;7(5):1257-63.

49. Van Tunen AJ, Hartman SA, Mur LA, Mol JN. Regulation of chalcone flavanone isomerase $(\mathrm{CHI})$ gene expression in Petunia hybrida: the use of alternative promoters in corolla, anthers and pollen. Plant Mol Biol. 1989;12(5):539-51.

50. Lu Y, Rausher MD. Evolutionary rate variation in anthocyanin pathway genes. Mol Biol Evol. 2003;20:1844-53.

51. Streisfeld MA, Liu D, Rausher MD. Predictable patterns of constraint among anthocyanin-regulating transcription factors in Ipomoea. New Phytol. 2011; 191(1):264-74

52. Yang Z, Wong WS, Nielsen R. Bayes empirical Bayes inference of amino acid sites under positive selection. Mol Biol Evol. 2005;22:1107-18.

53. Woolley S, Johnson J, Smith MJ, Crandall KA, McClellan DA. TreeSAAP: selection on amino acid properties using phylogenetic trees. Bioinformatics. 2003;19:671-2.

54. McClellan DA, McCracken KG. Estimating the influence of selection on the variable amino acid sites of the cytochrome $b$ protein functional domains. Mol Biol Evol. 2001;18:917-25.

55. Campanella JJ, Smalley JV, Dempsey ME. A phylogenetic examination of the primary anthocyanin production pathway of the Plantae. Bot Stud. 2014;55:10
56. Rausher MD. The evolution of flavonoids and their genes. In: Grotewold ER, editor. The science of flavonoids. New York: Springer; 2006. p. 175-211.

57. Shimada S, Takahashi K, Sato Y, Sakuta M. Dihydroflavonol 4-reductase cDNA from non-anthocyanin-producing species in the Caryophyllales. Plant Cell Physiol. 2004;45:1290-8.

58. Bovy A, Schijlen E, Hall RD. Metabolic engineering of flavonoids in tomato (Solanum lycopersicum): the potential for metabolomics. Metabolomics. 2007;3:399-412

59. Coburn RC, Griffin RH, Smith SD. Genetic basis of a rare floral mutant in an Andean species of Solanaceae. Am J Bot. 2015;102:264-72.

60. Wu CA, Streisfeld MA, Nutter LI, Cross KA. The genetic basis of a rare flower color polymorphism in Mimulus lewisii provides insight into the repeatability of evolution. Public Libr Sci One. 2013;8:e81173.

61. Smith SD, Rausher MD. Gene loss and parallel evolution contribute to species difference in flower color. Mol Biol Evol. 2011;28:2799-810.

62. Wessinger CA, Rausher MD. Predictability and irreversibility of genetic changes associated with flower color evolution in Penstemon barbatus. Evolution. 2014;68:1058-70

63. Wolfe AD, Randle CP, Datwyler SL, Morawetz JJ, Arguedas N, Diaz J. Phylogeny, taxonomic affinities, and biogeography of Penstemon (Plantaginaceae) based on ITS and cpDNA sequence data. Am J Bot. 2006:93:1699-713.

64. Wang X, Thomas SD, Zhang J. Relaxation of selective constraint and loss of function in the evolution of human bitter taste receptor genes. Hum Mol Genet. 2004;13:2671-8.

65. Beisswanger S, Stephan W. Evidence that strong positive selection drives neofunctionalization in the tandemly duplicated polyhomeotic genes in Drosophila. Proc Natl Acad Sci. 2008;105:5447-52.

66. Zhang J. Evolution by gene duplication: an update. Trends Ecol Evol. 2003;18:292-8.

67. Vitkup D, Kharchenko P, Wagner A. Influence of metabolic network structure and function on enzyme evolution. Genome Biol. 2006;7:R39.

68. Hahn MW, Conant GC, Wagner A. Molecular evolution in large genetic networks: does connectivity equal constraint? J Mol Evol. 2004;58:203-11.

69. Rausher MD, Miller RE, Tiffin P. Patterns of evolutionary rate variation among genes of the anthocyanin biosynthetic pathway. Mol Biol Evol. 1999;16:266-74.

70. Rausher MD, Lu Y, Meyer K. Variation in constraint versus positive selection as an explanation for evolutionary rate variation among anthocyanin genes. J Mol Evol. 2008;67(2):137-44.

71. Holton TA, Cornish EC. Genetics and biochemistry of anthocyanin biosynthesis. Plant Cell. 1995;7:1071.

72. Jez JM, Bowman ME, Dixon RA, Noel JP. Structure and mechanism of the evolutionarily unique plant enzyme chalcone isomerase. Nat Struct Mol Biol. 2000;7:786-91.

73. Cui J, Yuan X, Wang L, Jones G, Zhang S. Recent loss of vitamin C biosynthesis ability in bats. PloS One. 2011; doi:10.1371/journal.pone.0027114.

74. He X, Zhang J. Toward a molecular understanding of pleiotropy. Genetics. 2006;173:1885-91

75. Akashi H. Translational selection and yeast proteome evolution. Genetics. 2003;164(4):1291-303.

76. Drummond DA, Raval A, Wilke CO. A single determinant dominates the rate of yeast protein evolution. Mol Biol Evol. 2006;23(2):327-37.

77. Whittall JB, Voelckel C, Kliebenstein DJ, Hodges SA. Convergence, constraint and the role of gene expression during adaptive radiation: floral anthocyanins in Aquilegia. Mol Ecol. 2006:15(14):4645-57.

78. Yang Z. PAML 4: phylogenetic analysis by maximum likelihood. Mol Biol Evol. 2007:24:1586-91.

79. Yang Z. Likelihood ratio tests for detecting positive selection and application to primate lysozyme evolution. Mol Biol Evol. 1998;15:568-73.

80. Yang Z, Swanson WJ. Codon-substitution models to detect adaptive evolution that account for heterogeneous selective pressures among site classes. Mol Biol Evol. 2002;19(1):49-57.

81. Zhang J, Nielsen R, Yang Z. Evaluation of an improved branch-site likelihood method for detecting positive selection at the molecular level. Mol Biol Evol. 2005;22:2472-9.

82. Wong WS, Yang Z, Goldman N, Nielsen R. Accuracy and power of statistical methods for detecting adaptive evolution in protein coding sequences and for identifying positively selected sites. Genetics. 2004;168:1041-51.

83. McClellan DA, Palfreyman EJ, Smith MJ, Moss JL, Christensen RG, Sailsbery JK. Physicochemical evolution and molecular adaptation of the cetacean and artiodactyl cytochrome b proteins. Mol Biol Evol. 2005;22(3):437-55. 
84. Petit P, Granier T, d'Estaintot BL, Manigand C, Bathany K, Schmitter JM, Lauvergeat V, Hamdi S, Gallois B. Crystal structure of grape dihydroflavonol 4-reductase, a key enzyme in flavonoid biosynthesis. J Mol Biol. 2007:368(5):1345-57.

85. Guex N, Peitsch MC. SWISS-MODEL and the Swiss-Pdb Viewer: an environment for comparative protein modeling. Electrophoresis. 1997; 18(15):2714-23.

86. Muchhala N, Johnsen S, Smith SD. Competition for hummingbird pollination shapes flower color variation in Andean Solanaceae. Evolution. 2014;68:2275-86.

Submit your next manuscript to BioMed Central and we will help you at every step:

- We accept pre-submission inquiries

- Our selector tool helps you to find the most relevant journal

- We provide round the clock customer support

- Convenient online submission

- Thorough peer review

- Inclusion in PubMed and all major indexing services

- Maximum visibility for your research

Submit your manuscript at www.biomedcentral.com/submit
Biomed Central 